\title{
A NOTE ON THE IMAGES
}

Some of the images in this book present fully rendered reconstructions of buildings and sculptures that remain in only a fragmentary state. Although the process of creating them helped answer some important research questions, these images are presented here as illustrations. They are not included as evidence, and they should not be used as stand-alone arguments or evidence for these buildings. Alongside these images are detailed textual analyses of the fragments (and the potential knowledge they provide for buildings and sculpture) as well as images of the fragments themselves. This information provides the reader with a clear understanding of the state of the remains and of the degree to which extrapolation played a role in each reconstruction. Many elements in the reconstructions are open to substantially different interpretation, and the scholarly community should welcome other visual interpretations based on the evidence and on sound scholarship.

Like any reconstructions, these images will undoubtedly create an impression that will affect the study of the objects. A body of literature on such effects has grown impressively since the advent of 3 -D computer modeling and carries with it a debate about the choices one can make in reconstructions of such fragmentary evidence. I have used this literature to make determinations about how much detail to provide and when, in a way that I believe is both beneficial and clear to the reader. Where elements are completely unknown, they have been left in muted colors and without extraneous elaboration.

Although it has garnered extraordinary attention, in some ways, the use of 3-D technology in the creation of two-dimensional images (as is the case in this book) presents the reader with an illustration that is no different in its character than a sophisticated watercolor of Roman baths from the eighteenth century or a detailed drawing or pastel from the nineteenth or twentieth. All of these-drawings, watercolors, physical models, and virtual models of varying degrees of elaboration-present more of a building than exists and more of its reconstruction than can be conclusively determined from remaining evidence. I am aware that such images stick in the mind's eye and influence scholarship, but so too does the absence of such an image lead to misinformation and misunderstanding. The other extreme would be to illustrate only the fragmentary remains of the period and present no reconstructions or plans beyond the remains themselves and the words in this book. 
Yet by its fragmentation this presents an equally false picture of what the objects looked like when they were built. Furthermore, such piecemeal illustration has led many scholars to ignore the material and the period because its impressiveness and sophistication are muted by omission. I have chosen to use full illustrations to allow these great works of art and architecture a wider appreciation, and I have done so with a conscious effort to express the limitations of our knowledge. Because the images do not stand alone and because they are printed next to a textual resource, which explains in detail the limits of the state of the evidence, the reader is able to read this text and examine the endnotes to gauge how much of the reconstruction she or he wishes to credit. 